\title{
On the Origin of the Gamma-Ray Burst Redshift Distribution in the Early Universe
}

\author{
Shawqi Al Dallal1, Walid J. Azzam² \\ ${ }^{1}$ College of Graduate Studies and Research, Ahlia University, Manama, Bahrain \\ ${ }^{2}$ Department of Physics, College of Science, University of Bahrain, Sakhir, Bahrain \\ Email: shaldallal@gmail.com
}

Received 24 October 2014

\begin{abstract}
Gamma-ray bursts (GRBs) are the most powerful events in the universe, and are promising standard candles for cosmological studies. The data from NASA's Swift satellite reveal a distribution for the GRB number density that peaks at a redshift between 1 and 3 . In this paper, we classify GRBs based on their duration and discuss the origin of their progenitors. We shed light on the formation mechanism of supermassive black holes and massive stars at the early universe, and show how this process and other related events can lead to a relatively high number of GRBs that peak at high redshift.
\end{abstract}

\section{Keywords}

\section{Gamma-Ray Bursts, Black Holes, Early Universe}

\section{Introduction}

Gamma-ray bursts are extremely energetic explosions that hold great promise as cosmological probes of the early universe [1]. They were serendipitously discovered by the Vela satellites in the 1960s [2], and although a great deal of effort has gone into understanding their origin, the precise physical mechanism behind their formation remains elusive. Burst duration depends upon the mechanism involved and the nature of the progenitor and its environment. Bursts can last from tens of milliseconds to several minutes. A burst is usually followed by a longer-lived afterglow that appears at larger wavelengths [1]. Traditionally, GRBs have been classified, based on their duration, into long bursts (LGRBs) with $\mathrm{T}_{90}>2 \mathrm{~s}$, and short bursts (SGRBs) with $\mathrm{T}_{90}<2 \mathrm{~s}$, where $\mathrm{T}_{90}$ is the duration needed to accumulate $90 \%$ of the burst's fluence [3]. Recent observations reveal the existence of a third class of GRBs that exhibit a very short duration with $\mathrm{T}_{90}<0.1 \mathrm{~s}$ [4]. The durations' distributions for the different classes are very broad with a significant overlap, which renders the classification based on duration along a difficult task. The most powerful GRBs are characterized by a narrow beam of intense radiation released during a supernova or hypernova event, and are believed to be due to a collapse of a massive star followed by the formation of a compact object. Beaming effects are usually associated with LGRBs, and observations suggest significant variation in the jet angle ranging between 2 and 20 degrees [5]. The focusing of energy in a narrow jet causes the gamma rays emitted by most bursts to miss the Earth and never be detected. Short GRBs, on the other hand, are less collimated than long GRBs [6], and may sometimes be not collimated at all [7]. 
In the first part of this paper we introduce the collapsor model in which a massive star collapses to form a black hole. Subsequently, in the second part, we discuss the various models leading to SGRBs. This section is followed by a discussion of the possible mechanisms explaining the origin of the jets. The last section of the paper discusses the origin of certain energetic events that may lead to the formation GRBs in the early universe; this, in turn, may help explain the observed GRB redshift distribution.

\section{The Collapsor Model}

Observations of some long GRBs reveal a supernova connection. The most widely accepted mechanism involves the collapse of the core of an extremely massive, low-metallicity, rapidly rotating star to form a black hole [8]. The infalling matter swirls into a high density accretion disk and drives a pair of relativistic jets along the rotation axis [9]. The jets pummel through the stellar envelope and eventually interact with the stellar surface and radiate gamma rays. According to this model, the minimum angular momentum needed is basically the value associated with the last stable orbit around a black hole which, for non-rotating black holes is given by [10]:

$$
J=2(3)^{1 / 2} G M / c=4.6 \times 10^{16}\left[M_{B H} / 3 M_{\odot}\right] \mathrm{cm}^{2} / \mathrm{s}
$$

where $G$ is the universal gravitational constant, $c$ is the speed of light in vacuum, $M_{B H}$ is the mass of the black hole, and $M_{B H}$ is the solar mass. For rotating black holes with a $M_{\odot}$ parameter $a=1$, it is given by [11]

$$
J=2 G M /(3)^{1 / 2} c=1.5 \times 10^{16}\left[M_{B H} / 3 M_{\odot}\right] \mathrm{cm}^{2} / \mathrm{s}
$$

The afterglow emission appears at a longer wavelength, ranging from X-rays to radio waves. This process is relatively well understood and is usually explained in terms of energy or matter moving outward at nearly the speed of light. The collision of matter with the interstellar gas causes a relativistic shock-wave that propagates forward into interstellar space, while a second shock wave propagates back into the ejected matter. Strong local magnetic fields accelerate energetic electrons in the shock wave causing synchrotron emission across most of the electromagnetic spectrum [12] [13]. This model has been successful in explaining the behavior of many observed afterglows.

Alternative models for LGRBs replace the central black hole with a newly formed magnetar [14], keeping all other aspects of the model the same. LGRBs in our Milky Way galaxy are likely to come from the death of Wolf-Rayet stars.

\section{Origin of Short GRBs}

The collapsor model is unable to explain all types of GRBs. Observations reveal that some short duration GRBs may occur in systems in which there are no star formation processes. Several models have been advanced to explain the origin of SGRBs. The most favored model involves the merger of a binary system of two compact objects, whereby both stars spiral slowly toward each other, releasing energy via gravitational radiation [15] [16], until suddenly the tidal forces rip the prgenitors apart and the system collapses into a black hole. The infalling matter produces an accretion disk and releases a burst of energy, analogous to the collapsor model. Models proposed to explain the origin of SGRBs identify the binary compact objects as two neutron stars, a neutron star and a black hole, two black holes, or the evaporation of primordial black holes [17]-[20].

\section{Mechanism of Jet Formation}

The mechanism causing GRBs to convert energy into radiation is poorly understood, and there is no unequivocally accepted model describing this process [21]. However, recent observations of bright optical counterparts suggest that inverse Compton scattering is probably behind these events [22]. In this process, low energy photons are up-scattered to gamma-ray energies by relativistic electrons [23].

\section{Processes Unique to the Early Universe}

Current theoretical models suggest that the formation of GRBs is intimately related to black holes for all classes of GRBs. During the past few decades, several models have been advanced to explain the presence of massive or supermassive black holes in galaxy centers at redshifts corresponding to the era of the early universe. The 
crucial issue that remains is how the original black holes formed in the first place. In what follows, we shall investigate various possible channels that lead to the formation of black holes in the early universe.

\subsection{Population III Stars}

Black holes in the early universe were formed via various channels that depend on the local environment reigning in proto-galaxies. In the early universe, Population III stars are expected to form. These stars are massive, metal-free objects comprising the first generation of stars after the Big Bang. These stars are postulated to have formed in mini-halos with masses of the order of $10^{6} M_{\odot}$, and to have collapsed from the highest primordial density field. The fate of Population III stars depends primarily on their masses. The collapse of $40-140 M_{\odot}$ low metallicity stars is predicted to directly form a black hole [24]. When the mass of the Population III star is in the range 140 to $260 M_{\odot}$, the fate of the star is determined by the electron-positron pair production instability that leads to supernovae explosions [25] [26]. For yet higher masses ( $\left.>260 M_{\odot}\right)$, the fate of the star is determined by the photodisintegration instability. This process leads to the collapse of the star into a black hole [26] [27]. The final mass of the newly born black hole may reach at half the initial stellar mass [28].

\subsection{Gas Dynamics Instabilities}

Metal-free or metal poor proto-galaxies are efficient nurseries where black holes can form and grow. In these systems, supermassive black holes can also be formed directly out of a dense gas cloud [29]-[31]. However, enriched halos exhibit an efficient cooling process that favors fragmentation and star formation rather than direct black hole formation. In metal-free gas clouds that characterize the very first proto-galaxies, the collapse is expected to occur only in massive halos with virial temperatures $T_{\text {vir }}>10^{4} \mathrm{~K}$, where the formation of molecular hydrogen is inhibited, whereas atomic hydrogen becomes an efficient agent for cooling down the tenuous gas until it reaches $4000 \mathrm{~K}$ [32].

Local instabilities in a self-gravitating galactic disks can be calculated using the Toomre stability parameter defined as

$$
Q=\frac{C_{S} \kappa}{\pi G \Sigma}
$$

where $\Sigma$ is the surface mass density, $c_{\mathrm{s}}$ is the speed of sound, and $\kappa=\sqrt{2} V / R$ is the epicyclical frequency, and $V$ is the circular velocity of the disk of radius $R$. Gravitational instabilities occur when $Q$ approaches a critical value $Q_{\text {c. }}$ These instabilities might lead to mass infall rather than fragmentation and star formation, provided that destabilization of the system is kept below a threshold value where the inflow rate is below a critical threshold

$$
\dot{M}_{\max }=2 \alpha_{c} c_{s}^{3} / G
$$

Here, $\alpha_{c}$ is the viscosity parameter. The Toomre stability criterion requires that $T_{\text {vir }} \propto M_{h}^{2 / 3}$, where $M_{h}^{2 / 3}$ is the mass of the DM halo [33]. The upper limit of the mass that can contribute to MBH formation is determined by the mass and spin parameter of the halo.

\subsection{Collapse of Supermassive Stars}

Gas dynamic processes may lead to the formation of supermassive stars (SMS) that may collapse, under certain conditions, to form a massive black hole (MBH). Gas accumulated around the center of the halo can reach $10^{4}$ to $10^{6} M_{\odot}$ [32]. It has been shown that the rotation of SMS of a given mass, that are supported by radiation pressure, cannot halt the collapse, and thus MBHs is likely to form [34]. In systems where mass accumulation is fast enough, the outer layers of SMS are not thermally relaxed during much of the lifetime of a main sequence star [35]. These stars exhibit complex structures with a convective core surrounded by a convectively stable envelope containing most of the star's mass. Hydrogen burning in the core of these stars is relatively low, and continues throughout most of their massive stage. When hydrogen is exhausted, the SMS will contract and suffer catastrophic neutrino losses that lead to their collapse to a black hole of a few solar masses.

\section{Conclusion}

In this paper, the origin and the evolution of gamma-ray burst were presented. Furthermore, we have suggested 
that some events associated with the formation of galaxies are unique and occur only in the early universe. We propose that the formation of Population III stars or supermassive stars and their collapse into black holes is a viable source of gamma-ray bursts. Other processes resulting from gas dynamic instabilities may give rise, under certain conditions, to the formation of supermassive black holes. Black holes may also merge to form supermassive black holes at the centers of galaxies. Thus, the early universe is a fertile environment for the creation of black holes, and gamma-ray bursts may be a natural product of these black holes.

\section{References}

[1] Vedrenne, G. and Atteia, J.-L. (2009) Gamma-Ray Bursts: The Brightest Explosions in the Universe. Springer/Praxis Books.

[2] Klebesadel, et al., R.W. (1973) Observations of Gamma-Ray Bursts of Cosmic Origins. Astrophysical Journal, 182, L85-L88. http://dx.doi.org/10.1086/181225

[3] Kouveliotou, C., et al. (1993) Identification of Two Classes of Gamma-Ray Bursts. Astrophysical Journal, 413, L101-L104. http://dx.doi.org/10.1086/186969

[4] Cline, D.B., et al. (2011) Do Very Short Gamma-Ray Bursts Originate from Primordial Black Holes? Review. International Journal of Astronomy and Astrophysics, 1, 164-172. http://dx.doi.org/10.4236/ijaa.2011.13021

[5] Frail, D.A., et al. (2001) Beaming in Gamma-Ray Bursts: Evidence for a Standard Energy Reservoir. Astrophysical Journal Letters, 562, L557-L558. http://dx.doi.org/10.1086/338119

[6] Watson, D., et al. (2006) Are Short $\gamma$-Ray Bursts Collimated? GRB 050709, a Flare but No Break. Astronomy and Astrophysics, 454, L123-L126. http://dx.doi.org/10.1051/0004-6361:20065380

[7] Grupe, D., et al. (2006) Jet Breaks in Short Gamma-Ray Bursts. I: The Uncollimated Afterglow of GRB 050724. Astrophysical Journal, 653, 462.

[8] MacFadyen, A.I. and Woosley, S. (1999) Collapsars: Gamma-Ray Bursts and Explosions in 'Failed Supernovae’. Astrophysical Journal, 524, 262-289.

[9] Woosley, S.E. (1993) Gamma-Ray Bursts from Stellar Mass Accretion Disks around Black Holes. Astrophysical Journal, 405, 273-277. http://dx.doi.org/10.1086/172359

[10] Kouveliotou, C., et al., Eds. (2012) Gamma-Ray Bursts. Cambridge University Press, Cambridge. http://dx.doi.org/10.1017/CBO9780511980336

[11] Willott, C.J., et al. (2007) Four Quasars above Redshift 6 Discovered by Canada-France High-z Quasar Survey. Astron. J., 134, 2435-2450. http://dx.doi.org/10.1086/522962

[12] Sari, R., Piran, T. and Narayan, R. (1998) Spectra and Light Curves of Gamma-Ray Burst Afterglows. Astrophysical Journal Letters, 497, L17. http://dx.doi.org/10.1086/311269

[13] Nousek, J.A., et al. (2006) Evidence for a Canonical Gamma-Ray Burst Afterglow Light Curve in the Swift XRT Data. Astrophysical Journal, 642, 389-400. http://dx.doi.org/10.1086/500724

[14] Metzger, B., et al. (2007) AIP Conference Proceedings. SUPERNOVA 1987A: 20 YEARS AFTER: Supernovae and Gamma-Ray Bursters, 937, 521-525.

[15] Abbott, B., et al. (2007) Search for Gravitational Waves Associated with 39 Gamma-Ray Bursts Using Data from the Second, Third, and Fourth LIGO Runs. Physical Review D, 77, 062004. http://dx.doi.org/10.1103/PhysRevD.77.062004

[16] Kochanek, C.S. and Piran, T. (1993) Gravitational Waves and Gamma-Ray Bursts. Astrophysical Journal Letters, 417: L17-L23. http://dx.doi.org/10.1086/187083

[17] Vietri, M. and Stella, L. (1998) A Gamma-Ray Burst Model with Small Baryon Contamination. Astrophysical Journal Letters, 507, L45-L48. http://dx.doi.org/10.1086/311674

[18] MacFadyen, A.I. (2006) Late flares from GRBs—Clues about the Central Engine. AIP Conference Proceedings, 836, 48-53. http://dx.doi.org/10.1063/1.2207856

[19] Blinnikov, S., et al. (1984) Exploding Neutron Stars in Close Binaries. Soviet Astronomy Letters, 10, 177.

[20] Cline, D.B. (1996) Primordial Black-Hole Evaporation and the Quark-Gluon Phase Transition. Nuclear Physics A, 610, 500. http://dx.doi.org/10.1016/S0375-9474(96)00383-1

[21] Stern, B.E. and Poutanen, J. (2004) Gamma-Ray Bursts from Synchrotron Self-Compton Emission. Monthly Notices of the Royal Astronomical Society, 352, L35-L39. http://dx.doi.org/10.1111/j.1365-2966.2004.08163.x

[22] Racusin, J.L., et al. (2008) Broadband Observations of the Naked-Eye Gamma-Ray Burst GRB080319B. Nature, 455. 183-188. http://dx.doi.org/10.1038/nature07270 
[23] Wozniak, P.R., et al. (2009) Gamma-Ray Burst at the Extreme: The Naked-Eye Burst GRB 080319B. Astrophysical Journal, 691, 495-502. http://dx.doi.org/10.1038/nature07270

[24] Zhang, W., Woosley, S.E. and Heger, A. (2008) Fall Back and Black Hole Production in Massive Stars. Astrophys. J., 679, 639-654. http://dx.doi.org/10.1086/526404

[25] Barkat, Z., Rakavy, G. and Sack, N. (1967) Dynamics of Supernovae Explosion Resulting from Pain Formation. Phys. Rev. Lett., 18, 379-381. http://dx.doi.org/10.1103/PhysRevLett.18.379

[26] Bond, J.R., Arnett, W.D. and Carr, B.J. (1984) The Evolution and Fate of Very Massive Objects. Astrophys. J., 280, 825-847. http://dx.doi.org/10.1086/162057

[27] Woosley, S.E. and Weaver, T.A. (2010) The Physics of Supernova Explosions. Annual Rev. Astron. Astrophys., 24, 205-253. http://dx.doi.org/10.1146/annurev.aa.24.090186.001225

[28] Fryer, C.L., Woosley, S.E. and Heger, A. (2001) Pair-Instability Supernovae, Gravity Waves, and Gamma Ray Transients. Astrophy. J., 550, 372-382. http://dx.doi.org/10.1086/319719

[29] Haehnelt, M.G. and Rees, M.J. (1993) The Formation of Nuclei in Newly Formed Galaxies and the Evolution of the Quasar Population. Mon. Not. R. Astron. Soc., 263, 168-178. http://dx.doi.org/10.1093/mnras/263.1.168

[30] Loeb, A. and Rasio, F.A. (1994) Collapse of Primordial Gas Clouds and the Formation of Quasar Black Holes. Astrophys. J., 432, 52-61. http://dx.doi.org/10.1086/174548

[31] Lodato, G. and Natarajan, P. (2006) Supermassive Black Hole Formation during the Assembly of Pre-Galactic Discs. Mon. Not. R. Astron. Soc., 371, 1813-1823. http://dx.doi.org/10.1111/j.1365-2966.2006.10801.X

[32] Volonteri, M. (2010) Formation of Supermassive Black Holes. Astron. Astrophys Rev., 18, 279-315. http://dx.doi.org/10.1007/s00159-010-0029-x

[33] Miller, M.C. and Lauburg, V.M. (2009) Merger of Stellar-Mass Black Holels in Nuclear Star Clusters. Astrophys. J., 692, 917. http://dx.doi.org/10.1088/0004-637X/692/1/917

[34] Saijo, et al. (2002) Collapse of a Rotating Supermassive Star to a Supermassive Black Hole. Astrophys. J., 569, 349361. http://dx.doi.org/10.1086/339268

[35] Begelman, M.C. (2009) Evolution of Supermassive Stars as a Pathway to Black Hole Formation. arXiv:0910.4398 